\title{
A Model-Based Approach for Supporting Aspect-Oriented Development of Personal Information Management Systems
}

\author{
Caio Stein D'Agostini and Marco Winckler \\ ICS-IRIT, University Paul Sabatier \\ 118 route de Narbonne \\ 31062 Toulouse CEDEX 9, France \\ \{stein, winckler\}@irit.fr
}

\begin{abstract}
This paper is concerned by the development of tools for supporting personal information management over the Web; i.e. the storage and retrieval of personal information collected by users whilst interacting with Web applications. As personal information collections are unique to the user, it is very difficult to provide a uniform organization of information for everyone. Nonetheless, most personal information management systems (PIMS) will be concerned by similar aspects of information management such as information granularity, physical storage, policies for sharing information, versioning, etc. In this paper we analyze how information management aspects affect the development of PIMS. We start by identifying the relevant software aspects required to the development of PIMS. Then we describe how models featuring different aspects can be combined in a meta-model to build PIMS tools. This approach is illustrated by a case study.
\end{abstract}

Keywords: personal information management systems, aspect modeling, models composition.

\section{Introduction}

People naturally collect and store information that is relevant to their personal needs. In today's word people have an enormous quantity information on which depends the their daily lives; registration numbers with government services, addresses, telephones and e-mail addresses, banking information, etc. However, research has consistently shown that most of users have difficulties to remember where they placed their personal information and thus have difficulties in retrieving it (Jones \& Teevan, 2007). As the quantity of information a person possesses increases, users must develop additional mechanisms for organizing their information space.

Personal computers are often used with Personal Information Management (PIM) systems, as they allow people to collect items of information and store them outside their cognitive system (Malone, 1983). In the last years, users are more likely to interact with many applications and computers, thus causing fragmentation of their information space. Indeed, in order to manage their personal information, users frequently rely on multiple tools such as e-mail managers, agendas and file managers. 
The Fig. 1 illustrates how PIMS can be used to share personal information with other users and applications. When the users interact with services such their banks, online stores, or even other users, the PIMs provide the user a repository, an information space where they can store and re-find the information they need, such as their bank accounts, addresses to know where to deliver what gets bought, or contacts or photos they might want to share with their friends. However, fragmentation is just the tip of the iceberg. Users have to remember where their information is stored and also if it corresponds to the correct version and/or required format. Users also lose control of the information after it is shared, which can have serious consequences regarding the privacy and security. That is mostly in the context of the Web because many of the interactions with Web applications involve sharing of users' personal information.

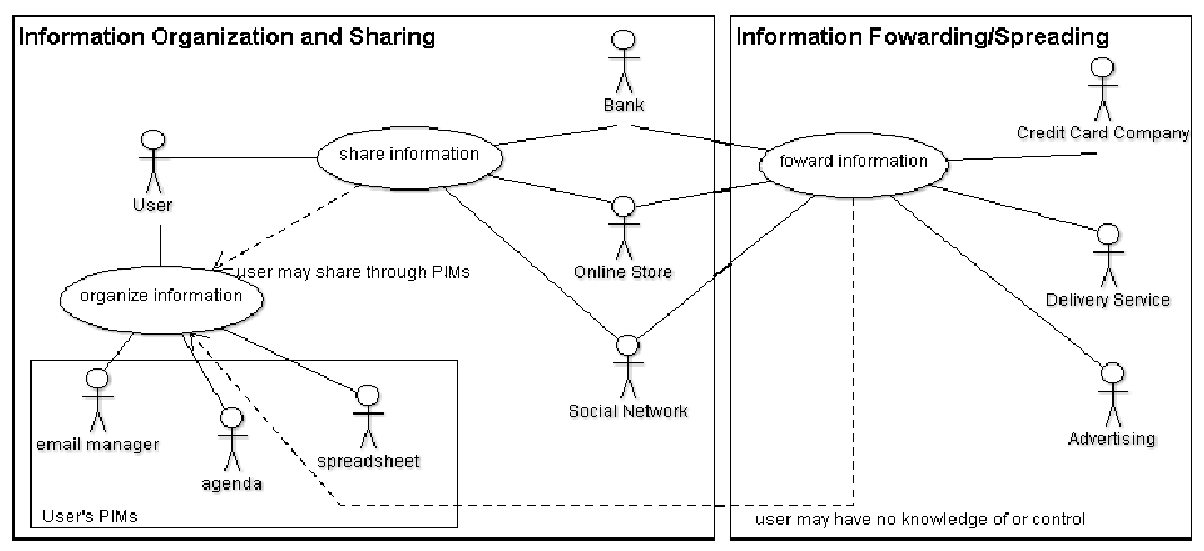

Fig. 1. PIMs and the use of personal information

Though personal information collections are unique to the user and the tasks accomplished with personal information vary, making it difficult to provide a uniform organization of the information space for everyone, most PIMS are concerned by similar aspects of information management such as information granularity, physical storage, disclosure, versioning, etc. Despite the fact these aspects concerns information management system in general, this paper is particularly interested in analyzing how such aspects affect the development of PIMS deployed over the Web.

This work is being developed for the PIMI project (Personal Information Management through Internet). The project already analyzed how users manage information and services and their expectations towards PIMs. (Scapin, 2011) analyses, through a mocked up system, the users' views on personal information management and their desires for flexibility to organize and classify the information, control of how it is shared and re-used.

Our goal on this paper is to try to understand how aspect modeling can help to build PIMS tools that fulfill the users' requirements for managing and sharing personal information. The paper is organized as follows: section 2 presents aspects regarding how users manage information; section 3 provides models for individual aspects and how they can be composed to build PIMS that fulfill different scenarios of use; section 4 describes and ongoing implementation of a PIMs based on our approach. 
The implementation took place simultaneously to the modeling and helped to better understand the different aspects. The rest of paper presents related and future work.

\section{Aspects Involved with Personal Information Management}

In this section introduces some aspects that affect the development of personal information management systems. These aspects are presented accordingly to the inner nature of personal information (section 2.1), the management of the information by the system (section 2.2) and the use made of the information (section 2.3). This section ends with an analysis of how existing tools support these aspects.

\subsection{Inner Nature of Personal Information}

Description and Classification: Information is only useful if the user can find it, meaning it has to be well organized. People organize their information based on i) categories that describe factual aspects (the information is an address, is a phone number, etc.) or ii) on episodic and situational aspects (the information is related to work, the information is related to the previous year's vacations, etc.) (Sauermann, 2007). The observations from (Sohn, 2011) indicate the importance of both classifications. The first type provides an easy method to find specific data, but users are not always interested on specific data; instead they may want to deal with information on an activity-based level, better fulfilled by the second strategy.

Granularity and Composition of the Information: Some information can be decomposed. The parts of the information can be, by themselves, meaningful information for the user. For example, when a user talks with its bank account manager, the account number probably gets shared. When giving the account information so that someone can transfer money the user shares different information, which is composed of the bank name, the agency number and account number. In the first case, the relevant grain of information is just the 'account number'. In the second situation, the grain is the 'bank account', which contains the 'account number'. The capacity to compose information relates to the its classification, as the user can compose information into groups, the same way folders are used to organize files on the computer.

Versioning: As time passes, information can change and the user has to keep track of the versions. This is an issue that also relates with the granularity of the information. Using the bank account example, the user may have its account transferred to another agency. Considering the bank account as the grain of information, the user still has the same bank account (it still refer to the same bank account in the real world), but its content has changed. By keeping track of versions the user can know who shares the current version and who shares the outdated one.

History of Use: Keeping track of the history of the information is important to help users keep track of how and when information were created, re-used or shared (Jensen, 2010) and who is involved in these actions. Taking the versioning example, there is an important event which is the change of agency. The user knows that on a 
given day the bank shared new information (the new agency) and the bank account got changed (new version). Knowing this history the user can know, for example, that all bank documents using the old agency refer to before this day.

\subsection{How Personal Information Is Handled by the System}

The following aspects affect the management of the personal information, but they are related to technical aspects, such as where the information is stored and from where it is accessed, or how it is represented.

Location of the Information: There are several possibilities on where to keep the information. The choice taken affects the information fragmentation:

- Store the information in only one information space (from several available spaces). This space can be the memory of a user's device or Web service. Every time it is necessary to use the information, it is accessed from that space. This option requires previous planning from the user on where to store the information (Sohn, 2011).

- Replicate the information in all of the spaces used by the user. This ensures that the information can be easily accessed. However, this solution may demands frequent synchronization to ensure all spaces have the same version of the information.

- Provide a unified view of the content on all of the spaces, as if there were only one (Dhumbumroong, 2011). The synchronization problem still persists and the solution assumes there are no incompatibilities when communicating with all the spaces.

- Store all the information in a single space. This solution provides one centralized point for storing the information. This space is used by the user and any other parties (users or services) that want to access the user's information. This solution supposes a generic solution capable of dealing with whatever requirement may arise.

Independently of the previous possibilities, parts of the information can be cached by applications, but this is an implementation choice which is not considered.

Versioning: Though versioning was already cited regarding the evolution of the information, it is also a technical aspect. If the user has the information stored replicated through different information spaces it might be difficult for the users to manage which version is stored on which space (Song, 2011). This may lead to problems when sharing an outdated information or overwriting the current version with an old one when trying to synchronize the different spaces.

Information Structure and Format: People can interpret information if they know what it is about and sometimes ignore that the same information can be represented in many ways. However information systems usually require data to conform to predefined schemas (Parent, 2000), as illustrated in Fig. 2. The figure shows three forms to represent the bank account; it can be identified by the bank name, agency and account number, it can be identified as IBAN (International Bank Account Number) or through a QR-Code which can encoded both information. The choice for one or the other may be influenced by legacy systems, standards, specific needs, etc.

Platform/Environment: Although each user has an individual organization and information can have multiple formats, the systems the users share their information with may not be that flexible. Systems might require that information follows certain schemas or may have restrictions to some formats. 


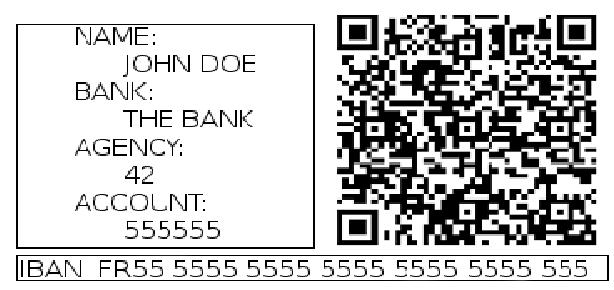

Fig. 2. Different structures and formats

Sharing and Control of the Shared Information: The sharing aspect involves giving access to specific information to others and knowing how the information is used after it is shared. Frequently when sharing information online it is necessary to accept a use agreement. Agreements are contracts that specify conditions of use, privacy and obligations of all involved parties (Meziane, 2010) and are the only control the user has over the shared information. However, once the user agrees with the contract, the information can be copied, re-shared, or the service can change its use agreement, all without knowledge or consent of the user. It is important, for reasons of privacy, security and trust to be able to revisit those conditions, so that the user can know if the information is being properly used.

\subsection{Regarding the Purpose of the Information}

Users might have different purposes when sharing personal information with other parties. This is not related to what is done to the information, but instead if the information is stored or not. This includes the following:

Information Is for Immediate Use: There are several services whose workings are not dependent on storing the user's information. Common examples are search engines or online maps; the user provides information as a parameter and receives results. Though the service may store the information, it is not necessary to do so.

Some Information Is Stored for Supporting an Activity: There are services that cannot be immediately executed, or which need to keep a history of the interactions with the user, even though the service is not an information service. This is the case of online shops that need to store personal information such as delivery addresses, credit card numbers, etc. Once the user shares the information with those systems, the direct control over the information is lost, at least partly (i.e.: the user may be able to change the delivery address, but the user has no control over previous orders).

Information Storage Is the Goal of the Service: There are services such as social networks, online file repositories, agendas, etc. whose purpose are store and manage information. Users usually have a personal account and control which information is provided and, if they want, they can remove or edit the information. Many of those services support information sharing (at least with other users of the same systems). But once the information is shared, the user also loses control over it. 


\subsection{Overview of Some Existing PIMS}

This section presents some systems for managing personal information and summarizes how they cope with the different aspects previously mentioned. It does not intend to provide and exhaustive list of tools, only to illustrate the diversity of tools available and the solution they provide to the issues presented. Table 1 list the different issues (1.Location of the information, 2.Platform, 3.Information Structure/Format, 4.Classification, 5.Versioning, 6.History of Use, 7.Sharing and control) and the approaches used by some tools to deal with them.

Table 1. Review of some existing PIMs

\begin{tabular}{|c|c|c|c|c|c|}
\hline & Dropbox & Evernote & $\begin{array}{c}\text { KDE } \\
\text { (Kontact, Akonadi, } \\
\text { Nepomuk) }\end{array}$ & PIMOnline & DeepaMehta \\
\hline 1 & $\begin{array}{l}\text { Web or local (infor- } \\
\text { mation is kept syn- } \\
\text { chronized) }\end{array}$ & Web & Local / remote servers & Web & Web \\
\hline 2 & $\begin{array}{l}\text { Browser, desktop, } \\
\text { mobile }\end{array}$ & $\begin{array}{l}\text { Browser, desk- } \\
\text { top, mobile }\end{array}$ & Desktop & Browser & Browser \\
\hline 3 & Files and folders & Notes and images & $\begin{array}{l}\text { Depends on the } \\
\text { application. Semantic } \\
\text { information can be } \\
\text { added with RDF. }\end{array}$ & $\begin{array}{l}\text { Calendar, contacts, } \\
\text { to-do list, notes }\end{array}$ & Files, text \\
\hline 4 & Folders & Tags & $\begin{array}{l}\text { Tags (using Nepo- } \\
\text { muk) or by type } \\
\text { (contacts, notes, } \\
\text { calendar) } \\
\end{array}$ & $\begin{array}{l}\text { Folders (for } \\
\text { notes). 'personal' } \\
\text { or 'business' (for } \\
\text { tasks) }\end{array}$ & $\begin{array}{l}\text { Association,/ compo- } \\
\text { sition, tags }\end{array}$ \\
\hline 5 & Yes & Yes & $\begin{array}{l}\text { No. It depends on the } \\
\text { individual PIMs }\end{array}$ & No & No \\
\hline 6 & $\begin{array}{l}\text { Timeline of what that } \\
\text { has happened in the } \\
\text { user's Dropbox }\end{array}$ & $\begin{array}{l}\text { No } \\
\text { It acts only as } \\
\text { storage }\end{array}$ & $\begin{array}{l}\text { No. It depends on the } \\
\text { individual PIMs }\end{array}$ & $\begin{array}{l}\text { No } \\
\text { It acts only as } \\
\text { storage }\end{array}$ & No \\
\hline 7 & $\begin{array}{l}\text { User can share } \\
\text { folders with other } \\
\text { Dropboxes or read- } \\
\text { only urls }\end{array}$ & $\begin{array}{l}\text { Shares the stored } \\
\text { information as a } \\
\text { link or e-mail }\end{array}$ & $\begin{array}{l}\text { Sharing between } \\
\text { applications that } \\
\text { implement the Ako- } \\
\text { nadi API }\end{array}$ & no & $\begin{array}{l}\text { Provides a shared } \\
\text { workspace. There is } \\
\text { no individual infor- } \\
\text { mation }\end{array}$ \\
\hline
\end{tabular}

Dropbox stores the information on the web and can be accessed as a web application or platform specific applications. Despite the timeline, it serves as file storage. It provides limited capabilities for classifying the stored content, organizing files into folders, which may not be adequate for all types of information. Evernote focuses on storing notes for personal re-use, not sharing. The notes are text fragments or images which can be describe with tags. Kontact acts only as an access point to the information managed by desktop applications, but it does not store them; the information can be classified with tags, using RDF triples (through Akonadi). DeepaMehta allows to compose and organize information as needed, but it is intended to manage information from shared projects, not individual information.

These examples show that, although there are tools that address the aspects and related problems presented on this work, the solutions are not all present simultaneously in a single application. 


\section{$3 \quad$ Modeling the Aspects}

This section presents a series of models that describe the aspects presented in the previous section. Moreover, it describes how individual models can be combined.

\subsection{Types of Service}

We begin to model the aspects associated to personal information management by identifying the different parties that may come to interact with the user's information.

A 'Party' is anyone or anything that uses the personal information. Though a party could be either a person or a system, when two users interact, they interact through services; they use their email services, their instant message systems, their social networks, etc. 'ConsumerOnly' are the parties that consume the information for immediate use, without persisting it, such as the search engines. A 'Collector' is a party that needs to store the information, and it can be of two types.

The 'InformationAsMean' collector are the ones that collect and stores information because they provide a service that cannot be immediately executed, or which demands to keep a history of past interactions. It is the case of online stores, who store the information to be used during the payment, preparation and shipping operations. And there is also the 'InformationAsEnd', which are the services whose explicit purpose are store and manage information.

Independently of the type, one party may use or store information differently from how it publicly promote its services. For example, even if the user has the option to delete information, the user has no way of knowing if the service actually removes the information or just "hides" it. This type of issue is related to reputation; whether a service can be trusted or not based on its historic of interaction with other users.

\subsection{The Sharing and Conditions of Use}

As previously described, while some times information is shared without establishing persistent links with the user, there are many situations where information is persisted and associated to the user, even if through the user's account.

On these situations it is very important to keep track of who has access to which information, and under which conditions. Knowing who uses the information is important so that the user knows who to notify when information changes, so everyone is kept up-to-date. It is also important to know how the information is going to be used, including whether or not it may be shared.

Sometimes services rely on third services to operate. For example, some on-line retail stores or auction services serve as proxies between the buyer and the product seller. Sometimes it is not clear to the user if the information given to the online store stays with the store, or if it is shared with the buyers (in case the user is selling) or the sellers (in case the user is buying).

Usually this is settled through contracts (disclaimers, conditions of use, privacy agreement, etc.) the user agrees with when subscribing to the service. The relation between information and how it is used is illustrated in Fig. 3. 


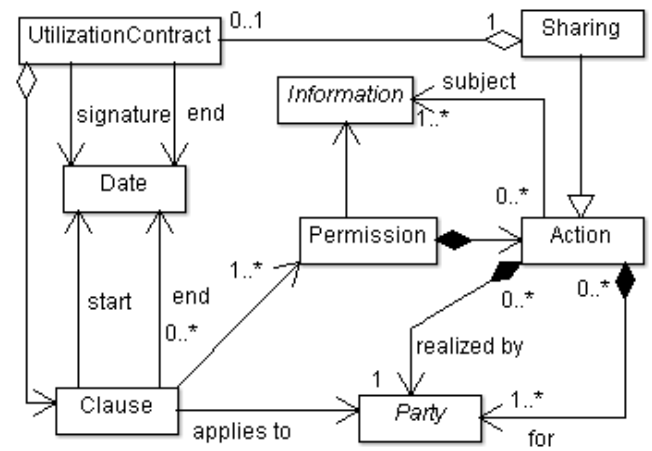

Fig. 3. Shared information and its utilization

Sharing information is an action that involves at least two parties, the one that has the information and the receiver(s). After the information is shared, its use may be governed by certain conditions ('UtilizationContract'). The contract lists which actions can (rights) or should (obligations) be performed by all involved parties, even if the user no longer has direct control over the information.

The conditions may not be the same for every party involved and they are also not restricted to the parties directly involved in the sharing (such as the case of the online store that forwards the user's information to the buyers or sellers).

\subsection{Keeping Track of Everything}

Though the contracts provide some security to the user by specifying what can be done with the information, users interact with several services on the Web. This means they keep sharing information with different parties, each time under a new contract. Keeping track of this history can be difficult for the user.

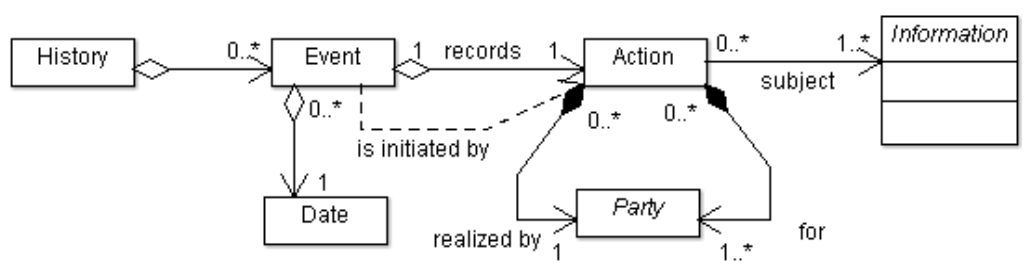

Fig. 4. Information's history of use

The history of use is a sequence of events. When information is shared, it is shared because of an event, such as buying a product on a store or adding a friend in a social network. But events can also relate to other actions, such as updating (e.g.; updating a profile on a social network) or removing information.

Thus, each user has a history regarding its personal information, composed of a series of events. Each event is associated with an action that took place on a certain moment ('Date'). The actions are performed by some party, to other party(ies) (which can be the same performing the action). The user can use any of those elements to recall information; e.g.: the user may remember the date the information was shared. 


\subsection{Different Formats and Versions}

Until this moment the model presented covered the types of parties that might use the information, how information is shared and how the user gradually creates a history of use of its personal information. It was assumed that information can be easily exchanged between different parties.

This part of the model focus not on the use of the information, but on its organization; how it is built, reused, represented and how it can change with time.

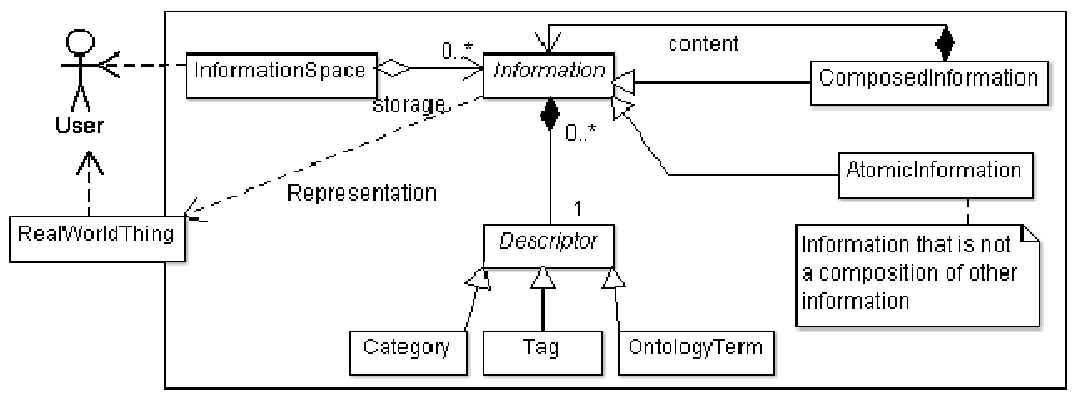

Fig. 5. Information description and composition

Fig. 5 models how users organize their information, considering they can store they information in an information space, without restrictions of form or structure.

One difficult is keeping information organized in such a way the user can find it, so that the information can be reused. The user can re-find information using its description, which can be provided through several means, such as categorization, tagging, semantic annotation using ontology terms, etc. The information can be composed or can be an atomic. Composed information re-uses other information. The atomic information is indivisible, and it can be a literal value, such as a text, or a file.

Once the description and composition is modeled, the model can be expanded to include restrictions on the format of the information, as shown in Fig. 6. Contrary to the scenario where the information space has no restrictions, usually information has to conform to schemas, which can vary between applications. One schema can define or limit how information can be composed, and it may also restrict the formats that information can be stored ('AtomicInformation'). The latter is a very important aspect, as it might limit the capacity of sharing information between different systems.

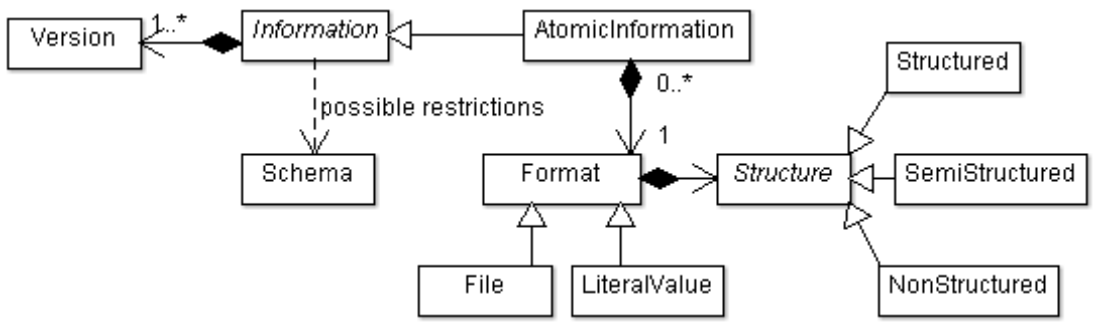

Fig. 6. Versioning and formats 


\subsection{Model Composition}

Once all the aspects have been modeled separately, it is necessary to put them all together and identify where their points of interaction are. Fig. 7 shows a slightly simplified complete model. The painted elements ('Information', 'Action', 'Party' and 'Sharing') are the one through which the different aspects interact.

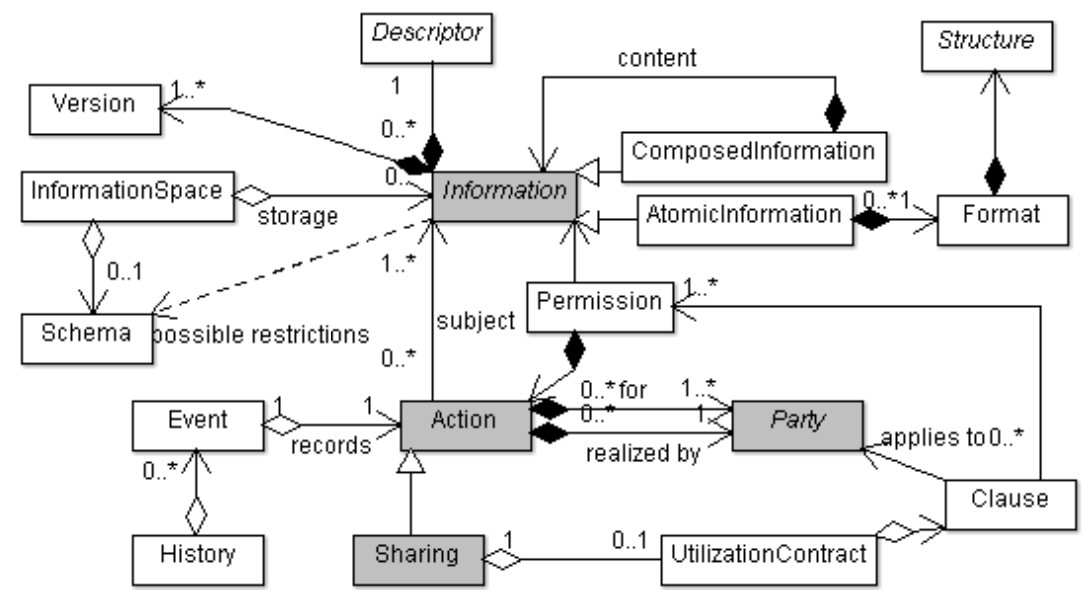

Fig. 7. The whole model and the connection points

The 'Information' is the center of the model, which is clear since the purpose of the model is to model the aspects related to personal information management. The 'Sharing' is a special type of action which allows the user to share and receive information from and with other parties. The history of who use the information and how it is used is a sequence of events that are constructed around the 'Action'.

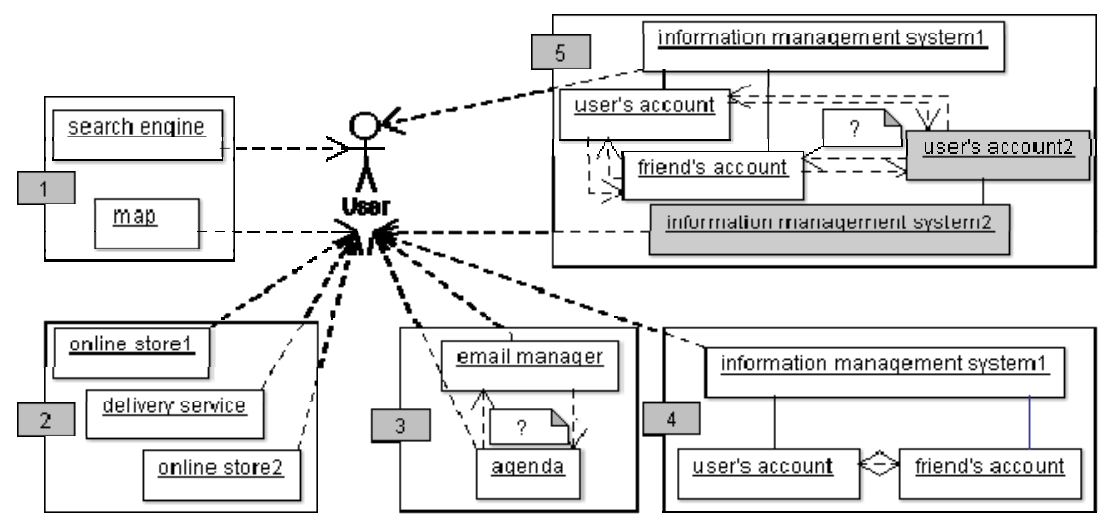

Fig. 8.Diversity of information uses and systems 
Fig. 8 shows several scenarios where personal information is used, and how different they can be depending on the different aspects. The scenario 1 shows services such as search engines and map tools that need information from the user to work, but do not store the information, neither they know who is using them. Scenario 2 shows a different type of situation where the services consuming the information need to trace the information to a specific user and also store the information. The same information can get stored in multiple services and once it gets stored the user no longer has control over it. Scenario 3 shows tools built to help managing personal information. Though the user has control over the information they store and can add or remove information freely, it is not always possible or easy to keep the information consistent between the different tools. Scenario 4 also shows a service built for managing personal information, but the same service is used by other users, each one with their own information. Since all information is managed by the same service, it is easy to share information with the other users. Scenario 5 describes a situation similar to 4 . It shows two similar systems, which handle the same type of information. However, there might be restriction and incompatibilities between them, such as differences in formats or schema. These differences may difficult managing the information between the information spaces from the systems.

\section{Implementation}

This section described the development of a prototype application. It is being developed iteratively, in parallel with the modeling of the aspects. It is used during the iterations to verify and adjust the models; for that, we use the bank account scenario, which is touched by all aspects and it is frequently experienced by people, thus it is well known.

So far, our prototype does not cover in details all of the aspects, as not all aspects can be approached in the implementation at the same time. For example, before keeping track of history it is necessary to provide support for operations (such as sharing), before providing sharing, it is necessary to implement the information model. More specifically, the prototype system can be included in the scenario 4 in Fig. 8. The system is a Web application for personal information management system where each user has its own personal information space. It was developed so that it can be accessed from mobile devices' Web browsers.

Fig. 9 shows the system's Web interface that gives access to the user's personal space. In the left of the image the personal space is populated with some information classified as 'Work' and/or 'Personal'. 'Work' is compose information containing the user's 'bank account' and 'work address' The right side depicts the interface after the 'bank account' information is opened, revealing two representations for the same information. The first is 'bank account' as a composition of bank, agency and account number; the second is a single atomic information, which is a document called RIB, used in France. 

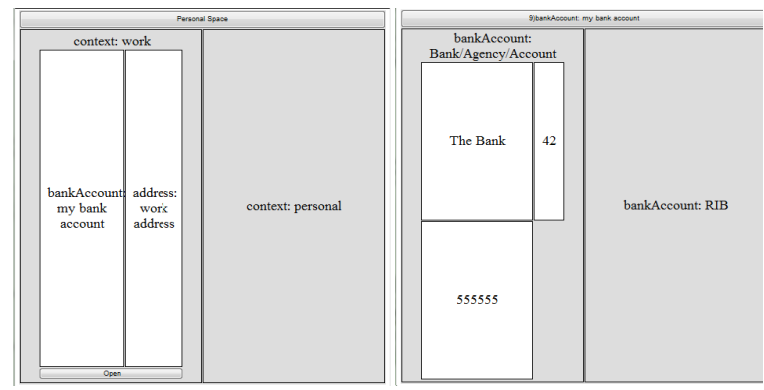

Fig. 9. Implementation of support for classification and composition

The implementation also allows the user to add new information, as shown in Fig. 10. The user gives a name to the information and can describe it further using keywords. The content can be a literal value or a file, which allows the user to store any information, despite of its format.

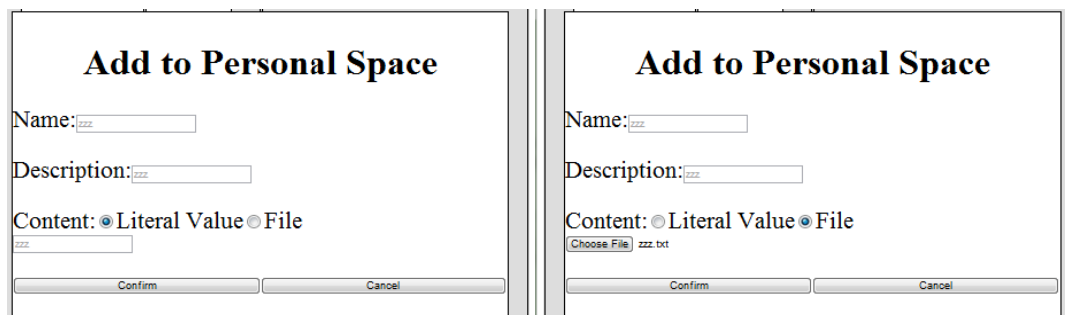

Fig. 10. Implementation of support for multiple formats of information

Finally, Fig. 11 demonstrates the sharing functionality. In the picture the user is sharing the whole content of the personal space. Once the information is shared it appears in the personal space of the other user, however only the original owner can edit it, since the contracts of use have not been implemented yet.

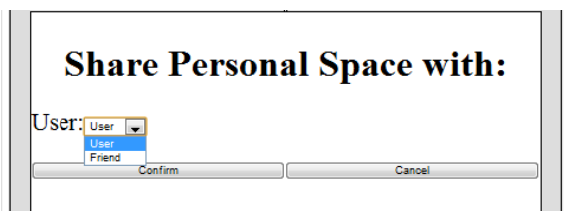

Fig. 11. Implementation of limited sharing

\section{$5 \quad$ Related Work}

So far, most of PIMs studies have mostly focused on very large data sets, such has the whole content of a user hard drive, and therefore has mainly concentrated on search/ retrieval issues; with some findings about the great variability in which people search their own information (Jones W. a., 2001). However, in more recent years some au- 
thors start to investigate the management of personal information over the Web (Voit, 2009) (Norrie, 2008) (Zhou, 2010). For example, Norrie (Norrie, 2008) proposes a complete architecture based on Web 2.0 technology enabling users to manage their personal records on the Web and synchronize them with other Web applications, in particular social networks. Notwithstanding, these efforts are mainly related to textual flat data and do not take into account interactive users tasks nor complex aspects of personal information management as discussed in this paper.

The benefits of decomposing the problems into different aspects when designing systems have already been pointed out (Barra, 2004). By identifying the aspects early on the development, the produced design becomes more reusable. The multiple representations for the same information were the first problem researched. The work (Parent, 2000) calls attention on the fact that the same information may adopt different representations depending on the intended use. Though work focus on geographic information systems (e.g.: in a detailed map a city is a composition of polygons and lines, on a smaller scale, the city may turn into a polygon, and on even smaller scale, only a point), the relevant problem is still how to work with multiple representations.

The work from (Dittrich, 2006) is centered on personal information management, more specifically on structured and semi-structured information. The work presents a model that hides the boundary between information inside and outside of a file. When implemented, the model provides the user with a single view that presents folders, files or the elements inside the files as pieces of information which are related one to the other, ignoring if he is viewing the contents of a specific file or folder.

The model proposed in (Kim, 2010) goes in the other direction. Instead of worrying with the content inside the files, it proposes to manage information through two spaces; a concept space and a document space. The documents in the document space are referenced by the 'items', which are instances of the concepts in the concept space. As long as the user describes the files, the information space has no need of knowing their contents. The user can perform keyword searches (assigning tags to the items) or faceted searches (through the concepts).

The PIMO model (Sauermann, 2007) is centered about the user's knowledge. The work argues that one limitation with many personal information management systems is that they burry the classification structures and their semantics in the applications, which makes difficult to personalize how the information is organized. Instead, PIMO proposes a model that use the user's personal knowledge (about people, roles, things, etc.), to create flexible categorizations which include episodic and situational information and can adapt to the user and to new circumstances of use.

Though until this moment most of the efforts were towards the issues with multiple formats, structures and classification of the information, the sharing aspect was also visited. The work on (Meziane, 2010) proposes a dynamic privacy model for web services. The model decomposes the privacy agreement into several elements. The elements cover not only the rights and obligations towards the information, but towards the agreement itself, so that it can be dynamically renegotiated with participation of all parts involved. 


\section{Conclusion and Future Work}

Personal information management is a daunting task as it might be influenced by many requirements raised by either by users that collect information as those users (or systems) that expect to consume information provides by personal information owners. Nonetheless, the identification of software aspects might help to choose features required for building PIMS.

In this paper we have discussed several aspects that might affect the development personal information systems. Whilst most of these aspects are not really new in information system in general, it is quite surprisingly they are barely supported by most of the existing tools, presented in Table 1.

Each aspect identified in this paper has been duly illustrated and modeled by scenarios that describe how they influence personal information management. Moreover, we have illustrated how these aspects can be combined to support different scenarios of PIMS. Such aspects may be considered for composing new applications or envisaging mapping mechanisms on existing tools.

We strongly believe that with the increasing use of Web applications, users will ultimately require more advance PIMS tools for help then to cope with such amount of personal information generated by such interactions. The results presented in this paper are rather preliminary but yet they contribute to understand how models can help to describe aspects related to PIMS and how to integrate them to build PIMS tools.

Future work will include the implementation of all aspects identified in this paper for covering all scenarios, which might include the integration and data exchange of PIMS with existing third party application. We also plan to refine the meta-model composition to integrate more fine-grained interaction of users with their personal data.

Acknowledgments. This work was performed under the French National Research Agency (ANR) project PIMI (Personal Information Management through Internet).

\section{References}

Barra, E.: An approach to Aspect Modelling with UML 2.0. In: Aspect-Oriented Modeling Workshop, AOM (2004)

Dhumbumroong, S.: Personal Cloud Filesystem: A distributed unification filesystem for. In: 2011 Eighth International Joint Conference on Computer Science and Software Engineering, JCSSE (2011)

Dittrich, J., Salles, M.: iDM: a unified and versatile data model for personal dataspace management. In: Proceedings of the International Conference on Very Large Data Bases (2006)

Jensen, C., et al.: The life and times of files and information: a study of desktop provenance. In: Proceedings of the 28th International Conference on Human Factors. ACM, New York (2010)

Jones, W.: Keeping found things found on the web. In: Proceedings of the Tenth International Conference on Information. ACM, New York (2001) 
Jones, W., Teevan, J.: Personal Information Management. University of Washington Press (2007)

Kim, J., et al.: Building a semantic representation for personal information. In: Proceedings of the 19th ACM International Conference on Information and Knowledge Management. ACM (2010)

Malone, T.W.: How Do People Organize Their Desks?: Implications for the Design of Office. ACM Trans. Inf. Syst., 99-112 (1983)

Meziane, H., Benbernou, S.: A dynamic privacy model for web services. In: Comput. Stand. Interfaces, Elsevier Science Publishers B.V., The Netherlands (2010)

Norrie, M.: PIM Meets Web 2.0. In: Li, Q., Spaccapietra, S., Yu, E., Olivé, A. (eds.) ER 2008. LNCS, vol. 5231, pp. 15-25. Springer, Heidelberg (2008)

Parent, C., et al.: MurMur: Database management of multiple representations. In: AAAI 2000 Workshop on Spatial and Temporal Granularity, Austin, Texas, USA (2000)

Sauermann, L., et al.: Pimo-a framework for representing personal information models. In: Proceedings of I-Semantics (2007)

Scapin, D., et al.: Personal Information Systems: User Views and Information Categorization. In: The Fourth International Conference on Advances in Human-oriented and Personalized Mechanisms, Technologies, and Services, CENTRIC 2011, ThinkMind, Barcelona (2011)

Sohn, T., et al.: Myngle: unifying and filtering web content for unplanned access between Multiple Personal Devices. In: Proceedings of the 13th International Conference on Ubiquitous Computing. ACM, New York (2011)

Song, G., Ling, C.: Users' Attitude and Strategies in Information Management With Multiple Computers. International Journal of Human-Computer Interaction (2011)

Voit, K., et al.: Why personal information management (pim) technologies are not widespread. In: Workshop on Personal Information Management 2009 (2009)

Zhou, D., et al.: Optimizing user interaction for Web-based mobile tasks. In: 19th International Conference on World Wide Web (WWW 2010). ACM, New York (2010) 